\title{
Diacronie
}

Studi di Storia Contemporanea

$N^{\circ} 16,4 \mid 2013$

Le monarchie nell'età dei nazionalismi

\section{Arcadi Espada, En nombre de Franco. Los héroes de la embajada de España en el Budapest nazi}

\section{Luca Costantini}

\section{(2) OpenEdition}

\section{Edizione digitale}

URL: http://journals.openedition.org/diacronie/967

DOI: $10.4000 /$ diacronie.967

ISSN: 2038-0925

\section{Editore}

Association culturelle Diacronie

\section{Notizia bibliografica digitale}

Luca Costantini, «Arcadi Espada, En nombre de Franco. Los héroes de la embajada de España en el Budapest nazi », Diacronie [Online], № 16, 4 | 2013, documento 16, Messo online il 01 décembre 2013, consultato il 24 septembre 2020. URL : http://journals.openedition.org/diacronie/967 ; DOI : https:// doi.org/10.4000/diacronie.967 


\section{Diacronie}

N. 16 | 4|2013 Le monarchie nell'età dei nazionalismi

$16 /$

\section{RECENSIONE:}

\section{Arcadi ESPADA, En nombre de Franco. Los héroes de la embajada de España en el Budapest nazi, Madrid, Espasa, 2013, 310 pp.} a cura di Luca COSTANTINI*

En nombre de Franco è un libro pubblicato nel 2013 dal giornalista, saggista ed opinionista spagnolo Arcadi Espada, per la casa editrice Espasa. Il volume, che nella versione spagnola conta 310 pagine, narra le vicende degli «eroi dell'ambasciata di Spagna nella Budapest nazista». Espada, che scrive per il quotidiano «El Mundo», è autore di testi come Diarios ${ }^{1}$ (vincitore del premio Espasa per la saggistica), Contra Catalunya ${ }^{2}$, Raval: del amor a los niños ${ }^{3}$, Notas para una biografía de Josep Pla ${ }^{4}$, Ebro/Orbe 5 , El terrorismo y sus etiquetas ${ }^{6}$, Samaranch: el deporte del poder 7.

Lo scenario della storia narrata nel volume En nombre de Franco è quello dell'occupazione nazista dell'Ungheria dal marzo del 1944, quando il governo dell'ammiraglio Horthy venne destituito per lasciar posto a un esecutivo guidato dal partito filonazista ungherese delle Croci Frecciate. Da quel momento si intensificarono i rastrellamenti e le deportazioni di migliaia di ebrei ungheresi verso i campi di sterminio nazisti in Polonia e Austria. In quei difficili mesi gli interessi spagnoli in Ungheria erano affidati al ministro Muguiro, reggente della legazione di Budapest, coadiuvato nel suo lavoro dal giovane console e diplomatico Ángel Sanz Briz. Quest'ultimo, che era al suo secondo incarico ufficiale dopo una parentesi a Il Cairo, divenne dal luglio del 1944, a seguito del rientro in Spagna di Muguiro, il principale responsabile della legazione spagnola di Budapest. Da quel momento entrava in possesso delle chiavi

\footnotetext{
${ }^{1}$ ESPADA, Arcadi, Diarios, Madrid, Espasa-Calpe, 2002.

2 ID., Contra Catalunya, Barcelona, Flor de Viento, 1997.

3 ID., Raval: del amor a los niños, Barcelona, Anagrama, 2000.

4 ID., Notas para una biografia de Josep Pla, Barcelona, Ediciones Omega, 2005.

5 ID., Ebro/Orbe, Barcelona, Tentadero, 2007.

6 ID., El terrorismo y sus etiquetas, Madrid, Espasa, 2007.

7 ID., Samaranch: el deporte del poder, Madrid, Espasa-Calpe, 1999.
} 
dell'ambasciata di un paese, la Spagna, dichiaratosi «neutrale» ma di fatto considerato "amico" della Germania nazista, nei confronti della quale, però, il giovane diplomatico non nutriva particolare simpatia.

I nazisti avevano occupato l'Ungheria per portare a termine la «Soluzione finale della questione ebraica» 8 . In quel frangente le lettere inviate dal diplomatico spagnolo alla moglie a Madrid rifletterono la preoccupazione per la sorte di amici e collaboratori in pericolo di vita. La deriva razziale del nazismo non era vista di buon occhio dallo spagnolo, che decise di opporvisi. Sanz Briz stilò migliaia di documenti e redasse, con l'aiuto della segretaria della legazione, la francese Madame Tourné, e del delegato agli affari legali, l'avvocato ungherese Zoltán Farkas, molte note dirette alle autorità magiare per il rispetto dei diritti degli ebrei posti sotto la protezione spagnola9. L'opera umanitaria dell'ambasciata spagnola di Budapest non fu però l'azione isolata del suo mandatario, ma fu il risultato di una serie di direttive provenienti dal Ministero degli Esteri di Madrid, espressione palese del volere di Francisco Franco, a cui Sanz Briz si attenne. La collaborazione di Franco al salvataggio degli ebrei è nota soprattutto per il caso degli ebrei francesi, che poterono valicare il confine dei Pirenei per giungere in Portogallo e di lì in America ${ }^{10}$; ma anche per quanto concerne gli ebrei ungheresi sembra che il Generalissimo si fosse adoperato per il buon esito dell'operazione ${ }^{11}$. In tal senso il testo di Arcadi Espada ha il merito di concentrarsi su un caso ancora poco noto, quello ungherese, a cui Franco guardava come salvacondotto per il regime in caso di vittoria alleata della guerra. Una scelta di Realpolitik che si intrecciava alle esigenze di legittimazione del regime franchista nell'ordine internazionale del dopoguerra, che oggi è possibile descrivere grazie alla scoperta di materiale d'archivio inedito inerente alle relazioni tra la politica franchista e l'Olocausto ${ }^{12}$.

\footnotetext{
${ }^{8} \mathrm{Si}$ vedano le note del processo ad Eichmann in ARENDT, Hannah, La banalità del male. Eichmann a Gerusalemme, Milano, Feltrinelli, 1998 [prima edizione 1964].

9 In principio gli ebrei protetti furono quelli d'origine safardita, cioè spagnola, discendenti degli esiliati del XV secolo durante il regno di Isabella di Castiglia e Ferdinando II d'Aragona. Ben presto, però, la protezione diplomatica si rivolse anche agli ebrei ungheresi, vincolati alla Spagna da ragioni familiari e commerciali (vere o presunte).

${ }_{10}$ LIPSCHITZ, Chaim U., Franco, Spain, the Jews and the Holocaust, New York, Ktav Publishing House, 1984.

11 CARCEDO, Diego, Un español frente al Holocausto, Madrid, Temas de Hoy, 2000.

${ }^{12}$ Per un quadro generale inerente la politica estera del regime franchista e la questione ebraica si rimanda a: CALVET, Josep, Las Montañas de la libertad, Madrid, Alianza editorial, 2010; ROTHER, Berndt, Franco y el Holocausto, Madrid, Marcial Pons, 2009; PAYNE, Stanley G., España, Alemania, la Segunda Guerra Mundial y el Holocausto, Madrid, La esfera de los libros, 2008; EIROA, Matilde, Las relaciones de Franco con Europa Centro-Oriental (1939-1955), Madrid, Ariel, 2001; SALINAS, David, España, los sefarditas y el Tercer Reich (1939-1945), Valladolid, Universidad de Valladolid, 1997; TUSELL, Javier, Franco, España y la segunda guerra mundial, Madrid, Temas de Hoy, 1995.
} 
Ma l'opera di Arcadi Espada non si limita ad analizzare la politica estera di Franco: prova ad andare oltre, associando ai fatti concreti un'interpretazione personale sul senso della ricerca storica. Nell'intervista all’ispanista ungherese Iván Harsány, quest'ultimo propone una chiave di lettura che sembra incidere profondamente sull'autore: «Ci sono cose che non sapremo mai. Ma sono poche» ${ }^{13}$. Ed è proprio a partire da questa interpretazione che Arcadi Espada decide di affrontare la seconda parte della sua ricerca, relativa alla storia di Giorgio Perlasca, il commerciante italiano che collaborò con l'ambasciata di Spagna nel salvataggio degli ebrei e che ne divenne a tal punto l'icona da ottenere l'appellativo di «Schindler italiano». Stando alla versione ufficiale, Giorgio Perlasca, reduce della guerra di Libia e della guerra civile spagnola, dopo l'8 settembre si rivolse all'ambasciata di Spagna per chiederne l'aiuto. In nome dell'apporto dato alla causa franchista del 1936-1939, gli venne consegnato un passaporto spagnolo, che dal dicembre del 1944 lo avrebbe spacciato per Sanz Briz, permettendogli di portare a termine l'opera umanitaria. Questa storia, divenuta nota al pubblico grazie al programma televisivo Mixer di Gianni Minoli14, andato in onda sulle reti Rai nel 1990, a cui seguirono il libro-intervista di Enrico Deaglio, e la fiction Rai del 2002, Perlasca. Un eroe italiano ${ }^{15}$, con Luca Zingaretti nel ruolo dello «Schindler italiano», non viene ritenuta plausibile da Arcadi Espada, che nella seconda metà del suo libro intavola un dialogo immaginario con l'italiano, culminato con l'accusa di avere falsato la realtà. Senza dubbio, precisa Espada, Perlasca collaborò con l'ambasciata spagnola nel salvataggio degli ebrei ungheresi, ma lo fece come comprimario e non come eroe solitario, così come ci è stato fatto credere. La tesi è che Perlasca non avrebbe potuto compiere tutto quello che dice di aver fatto senza il contributo essenziale di qualcun'altro: «Qualcuno doveva parlare ungherese, conoscere la politica ungherese ed essere un esperto conoscitore delle leggi diplomatiche ungheresi»16; qualcuno che Perlasca cercò di mettere in secondo piano, togliendolo di scena per assumerne i meriti: «A volte, è vero, non le resta altro rimedio che citarlo nel suo promemoria - conclude Espada rivolgendosi a Perlasca. Ma sempre accantonandolo.

\footnotetext{
${ }^{13}$ ESPADA, Arcadi, En nombre de Franco, Madrid, Espasa, 2013, p. 195 [traduzione a cura del Curatore].

${ }^{14}$ La puntata è disponibile anche su Youtube:

URL: < http://www.youtube.com/watch?v=PS5TyhaG_gk > [consultato il 5 ottobre 2013].

15 NEGRIN, Alberto, Giorgio Perlasca. Un eroe italiano, Rai Fiction-France 2-Hamster Productions-Sveriges Television-Palomar Endemol- Focusfilm Kft, Italia-Francia-SveziaUngheria, 2002, 197'.

${ }^{16}$ ESPADA, Arcadi, En nombre de Franco, Madrid, Espasa, 2013, p. 147.
} 
Un accompagnatore. Devo essere sincero: credo che l'accompagnatore fosse Lei. L’accompagnatore dell'avvocato Zoltán Farkas»17.

La storia narrata da Perlasca fu l'unica a giungere integra a distanza di anni e le notizie che possediamo su di lui sono frutto unicamente del suo racconto. Le sue memorie sono state pubblicate da Il Mulino nel libro L’impostore ${ }^{18}$, mentre un terzo volume, edito da Chiarelettere e scritto da Dalbert Hallenstein e Carlotta Zavattiero col titolo Giorgio Perlasca un italiano scomodo ${ }^{19}$, racconta la storia di Perlasca sottotitolandola: Vita e avventura di un fascista che da solo salvò migliaia di ebrei. Tutti questi libri considerano come vera una storia difficilmente confutabile, i cui protagonisti sono deceduti senza lasciare memorie scritte. Farkas morì nel gennaio del 1945, pochi giorni dopo l'entrata dei sovietici a Budapest, mentre la segretaria dell'ambasciata, la signora Tourné, si trasferì a Parigi nel 1956 e non proferì mai pubblica parola in merito agli avvenimenti dell'inverno del 1944. Ángel Sanz Briz fece, sì, alcune dichiarazioni, ma si attenne sempre al riserbo tipico del registro diplomatico: si tenga a mente che dal Ministero lo invitarono alla prudenza, nel timore che le voci sul salvataggio degli ebrei minassero le relazioni diplomatiche con i paesi arabi, indispensabili partner commerciali per il regime franchista. L'unica versione dettagliata fu, quindi, proprio quella narrata da Perlasca. Ma è proprio questa storia che Arcadi Espada vuole riscrivere, rimettendo al centro della scena Sanz Briz e l'avvocato Farkas, così come tutti coloro che collaborarono al salvataggio degli ebrei di Budapest. Il giudizio conclusivo dell'autore su Perlasca è severo: «C'è qualcosa, Signore, che non posso perdonarle, glielo anticipo. Il trattamento riservato nel suo racconto a due degli eroi dell'ambasciata di Spagna: l'avvocato Farkas e madame Tourné. Nessuno di loro scrisse un resoconto dell'inverno di Budapest. Però, al contrario di quanto avviene con Lei, sono i fatti e le testimonianze a parlare per loro. La verità è questa, asciutta: nella cronaca generale dell'inverno niente e nessuno parla di Giorgio Perlasca. Con un'eccezione importante: Lei medesimo ${ }^{20}$.

Si tratterebbe secondo Espada di ammettere la sostanziale falsità della storia raccontata da Perlasca. E la ragione di questa falsificazione andrebbe rinvenuta proprio nel promemoria che Perlasca inviò a Sanz Briz e al Ministero degli Esteri spagnoli poco dopo la fine della guerra. Lì spiegò le gesta svolte sotto l'insegna della bandiera rojigualda, ricordando che per la difesa degli interessi spagnoli avesse perduto ogni suo

\footnotetext{
17 Ibidem.

18 PERLASCA, Giorgio, L’impostore, Bologna, Il Mulino, 1997.

19 HALlENSTEIN, Dalbert, ZAVATTIERO, Carlotta, Giorgio Perlasca un italiano scomodo. Vita e avventura di un fascista che da solo salvò migliaia di ebrei, Milano, Chiarelettere, 2010.

${ }^{20}$ ESPADA, Arcadi, En nombre de Franco, Madrid, Espasa, 2013, p. 147.
} 
bene. Pur non chiedendo formalmente un rimborso per l'azione svolta, Espada sostiene che questa fosse, in ultima istanza, la ragione delle esagerazioni presenti nella storia tramandata dall'italiano. Col passare degli anni, poi, la storia di Perlasca ha ottenuto maggiore visibilità mediatica, al punto da coprire non solo l'opera di Sanz Briz ma anche quella degli altri protagonisti dell'ambasciata. Sebbene sia Perlasca che Sanz Briz siano stati insigniti dell'appellativo di "Giusti tra le nazioni” dall'istituto Yom Vashem, l'autore spagnolo sostiene che a livello d'immagine e di riconoscimento pubblico esista un profondo squilibrio tra i due "eroi". Con En nombre de Franco, dunque, Arcadi Espada propone che sia riconsegnato a tutti gli «eroi dell'ambasciata di Spagna nella Budapest nazista» il merito che realmente spetta loro. Così come il film di Spielberg su Schindler offrì una visione distorta della realtà (scrive Espada citando Álvaro Lozano: «A differenza di quanto accade nella realtà, quasi tutti si salvano» ${ }^{21}$ ), anche la storia di Perlasca sembra essere stata soggetta alla mitizzazione della fiction. La ricostruzione romanzata o cinematografica dei fatti costituisce in tal senso il limite della relazione tra la ricerca storica e la sua commercializzazione. Una relazione improduttiva e contraddittoria che l'autore prova così a descrivere:

Sto scrivendo questo libro grazie al film, e la memoria dei diplomatici europei che cercarono di salvare la vita delle comunità ebraiche assume importanza grazie a questo film. Si tratta del merito di Schindler's list e del cinema storico, in generale. L'altra faccia del merito sono i problemi che ogni scrittore scopre quando torna sui fatti narrati dal cinema e constata con disperazione che scrivere è correggere. [...] Perché è vero che il cinema sottrae molti fatti all'oblio; ma generalmente per trasferirli nell'altro pozzo profondo della fiction ${ }^{22}$.

$\mathrm{Al}$ di là delle considerazioni sul rapporto tra verità storica e fatti romanzati, il testo di Espada ha l'ambizione di inserirsi all'interno del dibattito storiografico, sia per quanto concerne la politica estera franchista negli anni Quaranta, sia rispetto al caso specifico degli eroi dell'ambasciata spagnola in Ungheria. La tesi e le conclusioni del volume poggiano su una ricerca archivistica che ha fatto emergere nuovi documenti e testimonianze, messi a disposizione del lettore nel sito web del libro. Lo stile narrativo è diretto e il registro giornalistico, peculiarità che hanno permesso all'autore di muoversi tra la descrizione dei fatti e le riflessioni personali. A guadagnarci è la lettura, che risulta piacevole e a tratti divertente. In Spagna questo volume è stato accolto con interesse da parte del pubblico e nel contesto italiano l'opera potrebbe aprire un

${ }^{21}$ ESPADA, Arcadi, En nombre de Franco, cit., p. 154.

22 Ibidem. 
dibattito sulla figura di Perlasca: una questione che sembra già aver interessato il «Corriere della Sera»23. In conclusione, si può dire che il libro di Arcadi Espada sia consigliabile a tutti coloro che apprezzano la narrazione storica e che amano riflettere sul senso della verità. Il racconto di Espada diventa in questo senso una sorta di viaggio che, da Madrid a Budapest, segue le tracce degli «eroi dell'ambasciata di Spagna», nella convinzione che sui fatti passati saranno sempre poche le cose che non riusciremo mai a sapere.

${ }^{23}$ NICASTRO, Andrea, Quello schiaffo al mito Perlasca, in «Corriere della Sera», 10 aprile 2013, URL:

<http://archiviostorico.corriere.it/2013/aprile/10/Quello_schiaffo_mito_Perlasca_co_o_2013 0410_36cd8bda-a1a1-11e2-8ad7-db752849c685.shtml> [consultato il 5 ottobre 2013]; NICASTRO, Andrea, Gli indignati del caso Perlasca, in «Corriere della Sera», 13 aprile 2013, p. 59, URL:

<http://archiviostorico.corriere.it/2013/aprile/13/Gli_indignati_del_caso_Perlasca_co_o_201 30413_3a682d6o-a3fc-11e2-a08c-6bca92797213.shtml > [consultato il 5 ottobre 2013]. 


\section{* L'autore}

Luca Costantini si è addottorato presso l'Università di Bologna e, in cotutela, con la Universidad Nacional de Educación a Distancia. È membro del Centro de Investigaciones Históricas de la Democracia Española e della Asociación de Historiadores del Presente. Collabora con le riviste «Ricerche di Storia Politica» e «Historia del Presente». Si occupa prevalentemente di partiti socialisti e socialdemocratici europei e di analisi politiche comparate in Italia e Spagna.

URL: < http://www.studistorici.com/progett/autori/\#Costantini >

\section{Per citare questo articolo:}

COSTANTINI, Luca, «Recensione: Arcadi ESPADA, En nombre de Franco. Los héroes de la embajada de España en el Budapest nazi, Madrid, Espasa, 2013, 310 pp.», Diacronie. Studi di Storia Contemporanea : Le monarchie nell'età dei nazionalismi, 29/12/2013,

URL: < http://www.studistorici.com/2013/12/29/costantini_numero_16/ >

Diacronie Studi di Storia Contemporanea 3 www.diacronie.it

Risorsa digitale indipendente a carattere storiografico. Uscita trimestrale.

redazione.diacronie@hotmail.it

Comitato di redazione: Marco Abram - Jacopo Bassi - Luca Bufarale - Alessandro Cattunar - Elisa Grandi - Deborah Paci - Fausto Pietrancosta - Matteo Tomasoni - Luca Zuccolo

Diritti: gli articoli di Diacronie. Studi di Storia Contemporanea sono pubblicati sotto licenza Creative Commons 2.5

Possono essere riprodotti a patto di non modificarne i contenuti e di non usarli per fini commerciali. La citazione di

estratti è comunque sempre autorizzata, nei limiti previsti dalla legge. 